\title{
TWO-PHOTON 3-D MAPPING OF TISSUE ENDOGENOUS FLUORESCENCE SPECIES BASED ON FLUORESCENCE EXCITATION AND EMISSION SPECTRA
}

\author{
LILY H. LAIHO*, THOMAS M. HANCEWICZ**, PETER D. KAPLAN**, PETER T. C. SO* \\ * Massachusetts Institute of Technology, Dept. of Mechanical Engineering, 77 Massachusetts \\ Avenue, Room 3-461A, Cambridge, Massachusetts 02139 \\ ** Unilever Research US Inc., Edgewater Laboratory, Edgewater, NJ 07020
}

Two-photon microscopy imaging of endogenous fluorescence has been shown to be a powerful method for the quantification of tissue structure and biochemistry. Autofluorescence imaging can provide useful tissue structural and biochemical information without fixation and staining procedures. It also offers the opportunity to develop non-invasive optical biopsy based on fluorescence contrast. Although autofluorescence is found in many different tissues, the endogenous fluorophores contributing to this autofluorescence and their distributions have not been completely identified. The analysis of tissue spectroscopic information with 3-D microscopic resolution can be used to assist in identifying these fluorophores. It has major advantages compared with typical tissue spectroscopy methods $[1,2,3]$. Previous works often examine the overall spectrum of a macroscopic sample or section, which is a mixture of many different fluorophores. The decomposition of this "mixed" spectra into individual components based on numerical methods such as principle component analysis is often a difficult task in complex tissues [4,5,6].

Our technique incorporates using both two-photon excitation for deep tissue imaging and image morphology guided analysis in order to extract information about the chemical components within the skin. This allows us to obtain structural information that provides additional a priori information for spectral decomposition. It isolates fluorescence signal that originates from microscopic volumes where there may be fewer independent fluorescent species. By reducing the mixing of different fluorescence species, the identity and contributions of each pure biochemical species can be more easily assessed.

This methodology is being applied to the study of human skin. In ex vivo specimens, in addition to the overall bulk emission spectrum of the skin (Figure 1), we have obtained layer-resolved emission spectra of the stratum corneum, stratum spinosum, basal layer, and dermis (Figure 2). From the image guided spectral analysis, it was determined that there are approximately five factors that contribute to most of the autofluorescence from human skin. Luminescence species identified include tryptophan, $\mathrm{NAD}(\mathrm{P}) \mathrm{H}$, melanin (or localizing species), and elastin that are autofluorescent and collagen matrix that contributes to a second harmonic signal.

References:

[1] R Na et al., Skin Research and Technology. 6 (2000) 112.

[2] H Zeng et al., Phys. Med. Biol. 38 (1993) 231.

[3] RR Alfano et al., Inst Electr Electron Eng J Quant Elect. QE-20 (1984) 1507.

[4] CY Wang et al., Photochemistry and Photobiology. 69 (1999) 471.

[5] H Tsurui et al., J of Histochemistry and Cytochemistry. 48 (2000) 653.

[6] Andrew, AJ, Hancewicz, TM. Applied Spectroscopy. 52 (1998) 797. 


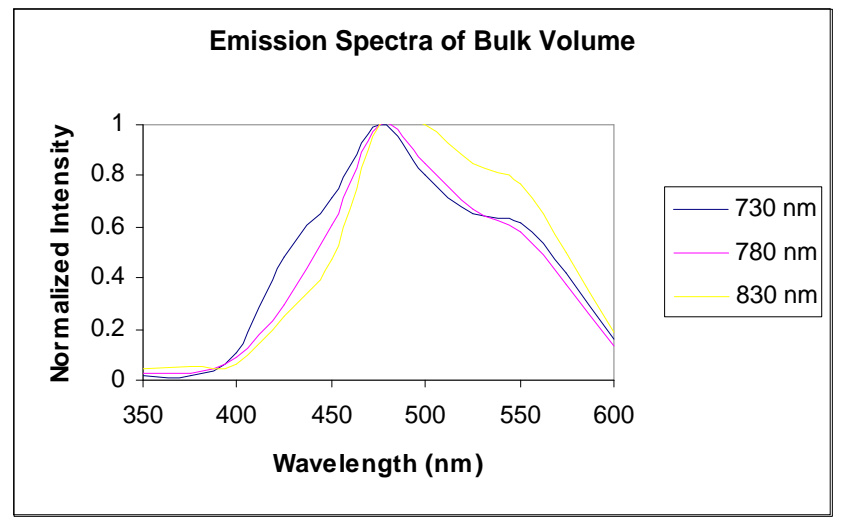

Fig. 1. Bulk emission spectra of human skin.

(a)
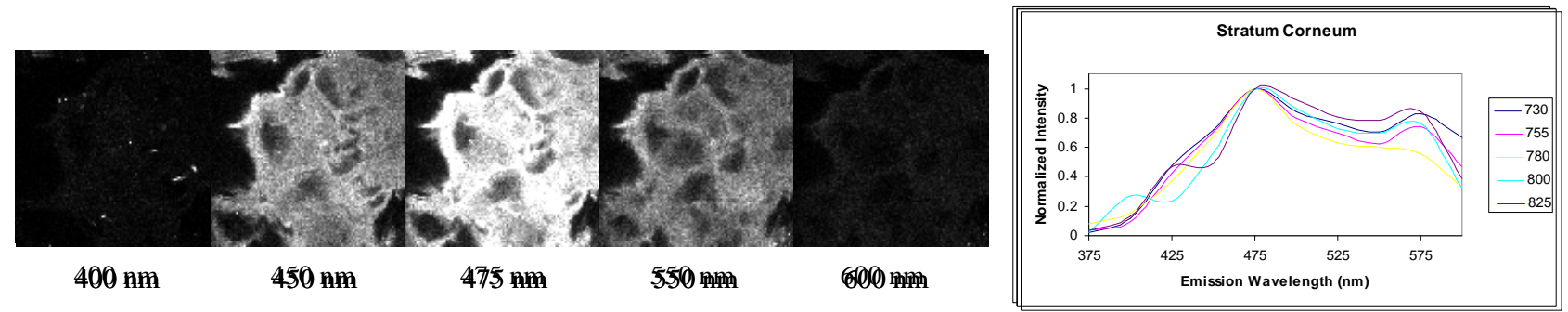

(b)
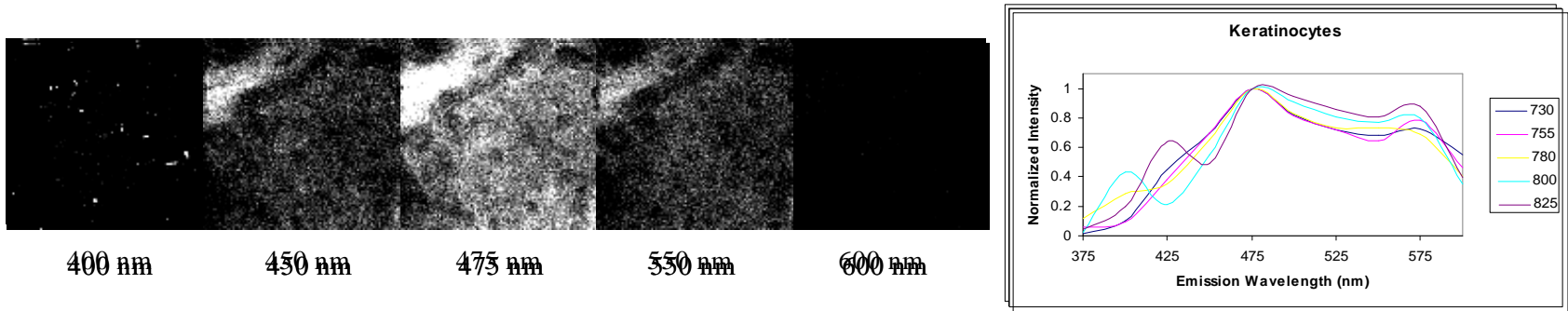

(c)
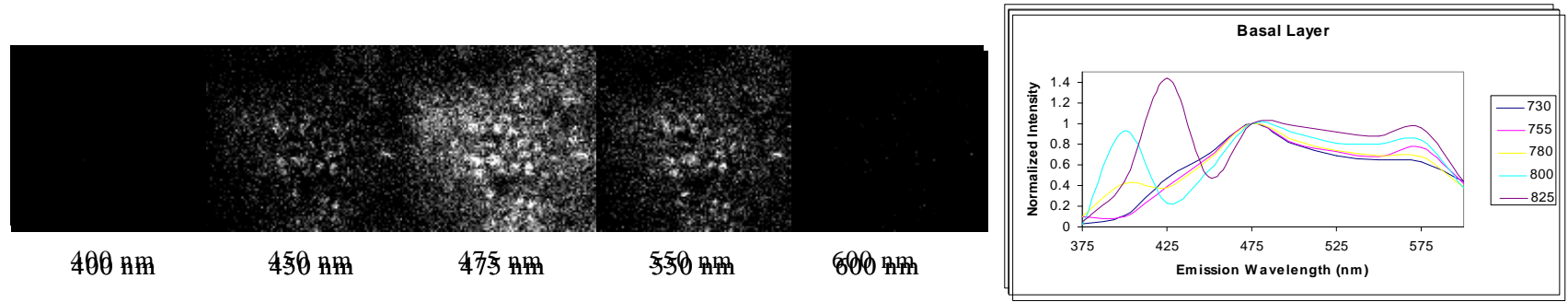

(d)
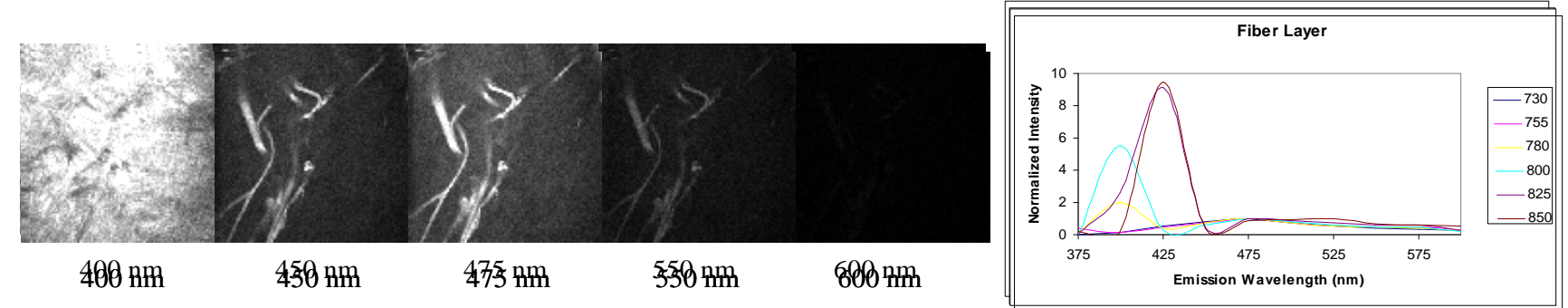

Fig. 2. Representative spectr al data and emission spectra for (a) stratum corneum, (b) stratum spinosum, (c) basal layer, and (d) dermis. 